\section{Revista Signos}

2010 / 43

Número Especial

Monográfico $\mathrm{N}^{\circ} 2$

311-326

\title{
Más allá del lenguaje sexista. Actos comunicativos en las relaciones afectivo-sexuales de los y las adolescentes
}

\author{
Oriol Ríos \\ Universitat Rovira i Virgili \\ España
}

\author{
Miranda Christou \\ University of Cyprus \\ Chipre
}

\begin{abstract}
Resumen: Uno de los problemas identificados para la cohesión social en Europa es el aumento de la violencia de género entre jóvenes. En este artículo analizamos los actos comunicativos en las relaciones afectivas y sexuales entre adolescentes, a partir de sus relatos sobre situaciones de interacción vividas o presenciadas. La información ha sido recogida a través de relatos de vida cotidiana y grupos de discusión comunicativos. El análisis de los actos comunicativos identifica el lenguaje verbal utilizado, pero también los gestos o el tono, la posición que ocupa el hablante o el atractivo que se le otorga en el grupo de iguales, la presión social, la atribución de deseo, etc., por los que unas mismas palabras, incluso unas mismas intenciones, pueden llegar a tener efectos diferentes. El estudio de las características de los actos comunicativos nos aporta criterios para identificar de forma más clara si en una relación existe acoso o libertad.
\end{abstract}

Palabras Clave: Actos comunicativos, adolescentes, violencia de género, relaciones afectivo-sexuales, atracción.

Recibido: 20-IV-2010 Aceptado: 9-VII-2010
Correspondencia: Oriol Ríos (joseoriol.rios@urv.cat). Departamento de Pedagogía. Facultat de Ciències de l'Educació i Psicología, Universitat Rovira i Virgili. Carretera de Valls s/n, 43007 Tarragona, España. 


\title{
Beyond sexism in language. Communicative acts in sexual and affective relationships among teenagers
}

\begin{abstract}
One of the barriers identified in terms of achieving social cohesion in Europe is the increase of gender violence amongst young people. In this article, we analyze the communicative acts that take place in young people's sexual-affective relationships through descriptions of the interaction they have experienced directly or have witnessed. The data for this analysis was gathered from daily life stories and communicative focus groups. The analysis of communicative acts identifies the verbal language used, but also gestures and tone of voice, as well as the speaker's social position. It also identifies the attractiveness attached to him or her within his or her peer group, social pressure, and the allocation of desire, etc. Differences in these factors can mean that the same word and even the same intentions can have different effects. Thus, the study of the characteristics of communicative acts provides us with criteria to clearly identify whether there is harassment or freedom in a relationship.
\end{abstract}

Key Words: Communicative acts, adolescents, gender violence, affective and sexual relationships, attraction.

\section{INTRODUCCIÓN}

La existencia de violencia de género en nuestra sociedad es una realidad frecuentemente visible a través de los medios de comunicación. Los datos en Europa sobre esta problemática así lo ejemplifican. España, por ejemplo, es una buena prueba de ello. En la encuesta más amplia sobre mujeres maltratadas realizada en este país, elaborada por el Instituto de la Mujer, se muestra que cerca de un $9 \%$ de mujeres entre 18 y 29 años fueron maltratadas a lo largo del año 2006. Por otro lado, los últimos datos sobre víctimas muestran una realidad aún más grave porque el porcentaje de muertes en el grupo de edad de 18 a 30 años supone el $29 \%$ del total (Instituto de la Mujer, 2006, 2010).

Acontecimientos recientes también han alarmado y conmocionado la luz pública respecto a problemáticas de esta índole. En enero de 2010 la joven irlandesa de 15 años, Phoebe Prince, se suicidaba en su casa de South Hadley, Massachusetts. Durante tres meses había sufrido un acoso feroz por parte de chicas y chicos de su instituto. Phoebe era una chica nueva en el instituto, y al llegar al centro empezó a salir con el chico más popular de la clase, el capitán del equipo de fútbol. Después de dejar la relación, un grupo de chicas, llamadas 'Chicas malas', y otros chicos empezaron a vejar y acosar a Phoebe. En el grupo de chicas se encontraba la ex novia del capitán. Posteriormente este mismo chico se empezó a relacionar con algunas de los miembros de la banda e iba difundiendo por el centro que había mantenido relaciones sexuales con Phoebe. Entre las actuaciones más habituales del grupo estaba la de insultarla en los diferentes 
espacios del centro. En casi todos la llamaban 'Zorra'. Después de la muerte de Phoebe, uno de los chicos acosadores publicó en la red social Facebook: "Misión cumplida” (Alandete, 2010).

Este caso ejemplifica cómo la utilización del lenguaje y la comunicación juega un papel central en las relaciones afectivas y sexuales de jóvenes y adolescentes: las etiquetas que se ponen a determinadas personas, el tipo de comunicación que mantienen los y las adolescentes a través de las redes virtuales, las consecuencias que esta comunicación desencadena, etc. De hecho las situaciones que se vivieron en el instituto americano evidencian el impacto y la violencia que ciertos usos del lenguaje pueden generar. De este modo, cuando se aborda este tipo de problemáticas es indispensable analizar las interacciones comunicativas y su influencia en el día a día de los centros educativos y espacios que comparten los chicos y chicas. En este artículo analizamos cómo los actos comunicativos existentes en las relaciones afectivo-sexuales de los y las adolescentes pueden promover la perpetuación o bien la superación de la violencia de género. Concretamente, se profundizará en los actos comunicativos que potencian relaciones donde existe igualdad y libertad (actos comunicativos dialógicos) o desigualdad y dominación (actos comunicativos de poder).

Este análisis se realiza en base a la investigación del Plan Nacional I+D+1' 'Actos comunicativos y superación de las desigualdades sociales en las relaciones de género' (CREA, 2006-2008) vinculada al proyecto integrado INCLUD-ED. 'Strategies for inclusion and social cohesion in Europe from education' (CREA, 2006-2011). El proyecto INCLUD-ED hace especial hincapié en la relación entre exclusión educativa y social que sufren cinco colectivos concretos: mujeres, personas con discapacidad, jóvenes, inmigrantes y minorías culturales. En este artículo se profundiza en una de las problemáticas que sufren las y los jóvenes: la violencia de género. En este sentido, en INCLUD-ED se realizó un informe preliminar sobre prácticas educativas relacionadas con el género que promovían o impedían el éxito escolar. En este análisis se incidía en la relevancia de considerar las aportaciones de la comunidad científica sobre las causas del fracaso y el éxito educativo en el diseño de los programas de prevención de la violencia de género y en los de educación sexual. Este análisis permitió ver cómo se estaban abordando problemáticas como la violencia de género entre jóvenes y adolescentes en el marco del contexto educativo (CREA, 2008). Siguiendo esta línea, el proyecto 'Actos comunicativos y superación de las desigualdades sociales en las relaciones de género' analiza con mayor profundidad esta problemática y cómo dicha violencia puede reproducirse o contribuir a superarse a través de los actos comunicativos.

El análisis realizado ha permitido identificar el impacto asociado a determinados actos comunicativos. Por una parte, existen aquellos actos comunicativos que favorecen relaciones cargadas de violencia al asociar atractivo a dominación y agresividad. Por otra parte, encontramos actos 
comunicativos en los que se vincula el atractivo y el deseo a personas con valores igualitarios y sin coerción. Esto implica una contribución importante en el estudio científico de las relaciones afectivo-sexuales, porque si bien es una temática que se ha investigado desde la perspectiva de las interacciones lingüísticas, hasta ahora no se ha profundizado desde el análisis de los actos comunicativos dialógicos o de poder.

El análisis de los actos comunicativos combina diferentes elementos: la influencia de la comunicación verbal y no-verbal, la diferencia entre las intenciones y las consecuencias de los actos y el contexto social de relaciones de poder entre los hablantes (Soler \& Ramis, 2008). De esta manera, como veremos, el análisis de los actos comunicativos aporta elementos que ayudan a identificar mejor las situaciones de acoso y, al mismo tiempo, promover estrategias que contribuyen a la prevención de la violencia de género.

En este artículo presentamos inicialmente un estado de la cuestión sobre las investigaciones y teorías existentes que realizan aproximaciones a la influencia de los actos comunicativos en las relaciones afectivo-sexuales. Seguidamente, nos centramos en la metodología llevada a cabo en el desarrollo del proyecto 'Actos comunicativos y superación de las desigualdades sociales en las relaciones de género’ que nos ha permitido llegar a los resultados que expondremos más adelante. Por último, dentro del apartado de resultados, nos centramos en aquellos elementos más relevantes que nos permiten identificar cómo se reproduce la violencia de género y cómo se puede superar a través los actos comunicativos.

\section{Estado de la cuestión: El impacto de los actos comunicativos en las relaciones afectivo- sexuales}

La literatura científica que aborda la influencia de los procesos de comunicación en las relaciones afectivo-sexuales muestra una diversidad amplia de explicaciones sobre el efecto del lenguaje verbal y no verbal en este tipo de relaciones y las problemáticas que se derivan de ellas, como la violencia de género (Stobbe, 2005; Klein, 2006; Boeringer, 1999). En la presente investigación se parte de dos conceptualizaciones fundamentales: los actos comunicativos (CREA, 2006-2008) y la socialización preventiva de la violencia de género (Duque, 2006; Gómez, 2004; Valls, Puigvert \& Duque, 2008).

La concepción de los actos comunicativos desarrollada por CREA (CREA, 2006-2008; Soler \& Ramis, 2008) aporta nuevos elementos al debate que Austin (1975), Searle (1999) y Habermas (1984) mantienen sobre los actos de habla. Estos autores han profundizado sobre la intencionalidad con que los sujetos emiten sus actos de habla y los efectos que pueden tener en los 
hablantes y oyentes. Pero desde la concepción de los actos comunicativos se tienen en cuenta no solo el lenguaje verbal, sino también el no verbal, incidiendo, sobre todo, en el papel que tienen las interacciones y el contexto en el momento que se produce la comunicación. En este sentido, creemos importante analizar estas situaciones considerando el impacto de la estructura social en las asunciones y el entendimiento que se dé entre los hablantes. De modo que habrá que considerar, por ejemplo, si existe una jerarquía en el momento de la comunicación ya que ello puede variar la interpretación que se haga de este acto comunicativo. A lo largo del artículo veremos la fuerte influencia que tiene este aspecto en las relaciones afectivosexuales.

Respecto de la teoría de la socialización preventiva de la violencia de género (Duque, 2006; Gómez, 2004; Valls et al., 2008), esta pone énfasis en el papel central que tiene el proceso de socialización en las relaciones afectivo-sexuales y en los modelos de atracción y elección, es decir, quiénes atraen, quiénes no, a quiénes se elige, a quiénes no, y por qué. Se parte del carácter social del amor y la importancia de diferentes componentes que influyen en su construcción. Uno de estos componentes es la forma de hablar y el lenguaje que utilizamos ante estas situaciones. Desde esta perspectiva se considera que a través del diálogo es posible influir en los procesos de socialización y modificar los gustos y preferencias que inciden en las elecciones en las relaciones afectivo-sexuales. Gómez (2004) y Duque (2006) analizan qué tipo de relaciones afectivo-sexuales tienen los jóvenes y adolescentes en la actualidad y cómo en muchas de ellas se establece una vinculación clara entre violencia y atractivo. Al mismo tiempo, estos autores ejemplifican la ausencia de deseo vinculada a las relaciones donde existe un modelo masculino basado en valores igualitarios. Ambos autores argumentan que esta vinculación se debe a los procesos de socialización a los que están expuestos los y las adolescentes y jóvenes, y cómo dichos procesos a menudo se fundamentan en actos comunicativos de poder, es decir, basados en interacciones de dominación o abuso. Para romper con esta dinámica, como se ha argumentado anteriormente, proponen un proceso de socialización forjado en el diálogo y la reflexión que permita desvincular la violencia con el deseo y, al mismo tiempo, dotar de atractivo aspectos como la igualdad y el respeto. Es de esta forma como Gómez (2004) y Duque (2006) consideran que se puede trabajar para prevenir relaciones que lleven a la violencia de género, a través por ejemplo de actos comunicativos dialógicos que relacionen igualdad con el deseo y el atractivo.

La literatura científica sobre la influencia de los actos comunicativos en las relaciones afectivosexuales de personas adultas, jóvenes y adolescentes muestra diferentes tendencias. Estas tendencias se centran, sobre todo, en el análisis del discurso de hombres y mujeres y en la influencia del contexto social en la comunicación interpersonal. En este sentido, la literatura 
insiste en la influencia de la construcción de un lenguaje sexista en diferentes contextos a través del cual se discrimina de diferentes formas a las mujeres (Klein, 2006; Stobbe, 2005; Boeringer, 1999). Pero existe una ausencia de estudios que analicen cómo el lenguaje, ya sea verbal o no verbal, tiene una incidencia en la construcción de los modelos de atractivo y en la elección en las relaciones afectivo-sexuales.

Así, desde el análisis del discurso existen investigaciones que subrayan el proceso de construcción de un discurso dominante donde el hombre ejerce una fuerte discriminación hacia la mujer (Klein, 2006; Stobbe, 2005). En estos estudios se constata la reproducción de unas imágenes despectivas hacia la mujer que llevan a la construcción de unos actos comunicativos que perpetúan su situación de desigualdad en ámbitos como el trabajo y que pueden tener como efecto la violencia de género.

Por otro lado, las investigaciones que han analizado la construcción del lenguaje en determinados contextos con una presencia importante de hombres exponen una serie de riesgos que estos espacios implican para las mujeres. Por ejemplo, poniendo el énfasis en las comunidades de estudiantes, en las fraternidades o grupos deportivos, se observa que en estos espacios se va articulando un lenguaje despectivo hacia la mujer que degrada su imagen. Esta dinámica puede derivar en prácticas de coerción sexual (Boeringer, 1999; Klein, 2006), ya que el lenguaje en este tipo de espacios y situaciones no juega un papel neutro en el desarrollo de las actitudes por parte de los chicos y chicas. Todo lo contrario, tiene un impacto en las relaciones entre géneros que puede acabar derivando en relaciones de extrema violencia hacia las mujeres. Finalmente, estos estudios concluyen que la masculinización de estos espacios favorece que se reproduzca el modelo de masculinidad hegemónica donde los hombres adquieren actitudes y formas de actuar basadas en la violencia, la agresividad y el abuso de poder (Connell, 2005).

En esta línea también se sitúan algunas investigaciones que apuntan a que los procesos de socialización de género determinan el tipo de comunicación que establecen hombres y mujeres (Giordano, Longmore \& Manning, 2006; MacGeorge, Graves, Feng \& Burleson, 2004). Esto implica diferenciar entre dos formas distintas de comunicación en función del género. Por un lado, se identifica que los hombres tienen una forma de comunicarse más cercana a la dominación, mientras que la de las mujeres se acerca más a la compasión. Otros aspectos que se subrayan hacen referencia a como ellos evaden a menudo conversaciones vinculadas a la intimidad mientras que ellas tienden a manifestar sus problemas y a compartirlos con sus compañeras. Desde otra perspectiva más vinculada a la influencia de los procesos de comunicación como forma de superación de la violencia de género, se apunta que el diálogo que las mujeres establecen con miembros de su entorno las 'empodera' para poder reconocer situaciones de maltrato y abandonarlas (Hage, 2006). 
Los resultados que se presentan en el artículo aportan nuevo conocimiento sobre la temática analizada y van más allá de la identificación que existe en la literatura científica sobre el lenguaje sexista en las relaciones afectivo-sexuales. En los siguientes apartados se expondrán estos resultados subrayando su contribución al análisis de los actos comunicativos y su influencia en las relaciones afectivo-sexuales de las y los adolescentes.

\section{Metodología}

La metodología que se ha utilizado en la investigación en la que se basa este artículo se sitúa en el paradigma comunicativo crítico (Flecha \& Gómez, 2004). Este tipo de metodología conlleva la implicación, en plano de igualdad, de los actores a los que va dirigida la investigación. De esta manera, el conocimiento se genera a partir del diálogo que se establece entre ambas partes; desde el equipo investigador se aportan los datos y teorías que la comunidad científica posee sobre esa temática mientras que los actores sociales contribuyen con sus experiencias e interpretaciones sobre la realidad social que se está estudiando. Este diálogo debe fundamentarse en argumentos que posibiliten llegar a un consenso acerca de las interpretaciones científicas que se derivan de los análisis realizados.

En el caso del proyecto de 'Actos comunicativos y superación de las desigualdades sociales en las relaciones de género' se ha incluido la participación de chicas y chicos jóvenes y adolescentes en diferentes momentos del proceso investigador. Esta participación se ha concretado fundamentalmente a partir de la configuración de un Consejo Asesor formado por adolescentes y jóvenes. La inclusión dentro de la investigación de este órgano da validez a los resultados y permite orientar sus conclusiones con el objetivo de superar problemáticas como las relaciones de género desiguales o la violencia de género. El Consejo Asesor de esta investigación estaba formado por dos chicos y dos chicas de 17 a 20 años de edad, una de ellas, perteneciente a la comunidad gitana.

A lo largo de la investigación se mantuvieron diferentes reuniones con los miembros del Consejo para contrastar los resultados que se iban obteniendo. Ello permitió corroborar, por ejemplo, la existencia de interacciones comunicativas en las que los chicos presionan a las chicas para mantener relaciones sexuales, o también el efecto de los comentarios del grupo de iguales en este tipo de situaciones. En este sentido los participantes en el Consejo subrayaron la trascendencia que tiene esta presión en muchas relaciones porque en ocasiones se traduce en situaciones donde no existe un deseo explícito por mantenerlas. Ello conduce a entablar relaciones afectivo-sexuales que son insatisfactorias. 
Asimismo, la inclusión de la voz de los y las adolescentes en la investigación empírica se concretó también a través de su participación en el trabajo de campo. Para ello se realizaron un total de catorce técnicas de recogida de datos cualitativas con orientación comunicativa. Se realizaron diez relatos comunicativos de vida cotidiana a chicas de entre 15 a 18 años, dos grupos de discusión comunicativos con chicas de 15 a 16 años y dos con chicos de estas mismas edades. Estas técnicas se realizaron en cinco institutos de secundaria de tres comunidades autónomas y se contó con la autorización oficial de las familias, profesorado y el propio alumnado. A estas técnicas se añadieron diez entrevistas en profundidad a profesionales implicados en la prevención de la violencia de género, de modo que se contactó con responsables políticos, técnicos y miembros de entidades vinculadas a este ámbito.

\section{Análisis de los actos comunicativos entre adolescentes y su impacto en las relaciones afectivo-sexuales}

Los resultados del análisis llevado a cabo muestran claramente cómo en los actos comunicativos de las y los jóvenes y adolescentes surgen nuevas problemáticas y situaciones vinculadas a las relaciones afectivo-sexuales que van más allá de la reproducción del lenguaje sexista. El análisis de los actos comunicativos nos ha permitido identificar la existencia de un lenguaje de la ética y de un lenguaje del deseo en las interacciones que los y las adolescentes establecen en torno a las relaciones afectivo-sexuales. El uso de los diferentes lenguajes y la interpretación que los y las adolescentes van construyendo de la realidad, ha tenido un impacto decisivo en el tipo de relaciones que eligen mantener: bien basadas en la dominación y sin libertad, o bien en el diálogo y libres de violencia. Algunos de los resultados obtenidos constatan la existencia de actos comunicativos de poder en las relaciones afectivo-sexuales que promueven interacciones de poder que dan lugar a relaciones de coacción, incluso violentas. Por otro lado, la incorporación de actos comunicativos dialógicos, basados en la solidaridad y en la sinceridad, está contribuyendo a superar las situaciones de abuso y prevenir la violencia de género.

\subsection{Lenguaje de la ética vs. lenguaje del deseo}

El primer aspecto que queremos subrayar es el lenguaje que los y las adolescentes utilizan para hablar de sus relaciones afectivo-sexuales y que se concreta en determinados actos comunicativos. La manera cómo las y los jóvenes muestran interés por determinadas relaciones o personas se transmite a través de actos comunicativos en los que se incluyen componentes verbales y no verbales concretos. En este sentido la investigación realizada ha podido observar que estos actos comunicativos se van definiendo a través de un lenguaje basado en el deseo donde las 
palabras, expresiones y actitudes corporales vinculan el atractivo con la excitación hacia la violencia (Flecha, 2008; Puigvert, 2008). En algunos de estos actos comunicativos se observa, en cambio, que para explicitar la bondad, es decir, los valores igualitarios que emanan ciertas personas, los y las adolescentes usan un lenguaje relacionado con la ética donde no se atribuye ningún tipo de atractivo a chicos y chicas que presentan este tipo de valores. De manera que se constata, que más allá del sexismo en el lenguaje, el análisis de los actos comunicativos permite identificar cómo a través del lenguaje los y las jóvenes y adolescentes asocian deseo -o hablan con deseo- a relaciones donde hay violencia y/o dominación, mientras que las que se relacionan con la bondad, se asocian con la ética (lenguaje de la ética). Como veremos más adelante, el efecto directo de estas modalidades de lenguaje puede ser la promoción de relaciones basadas en la dominación y el desprecio.

\section{“¿Por qué atraen si en cambio tratan mal a las chicas?}

Porque de entrada suelen ser así como chulitos, interesantes, que te fijas en ellos. Pero si eres un poco lista ya los dejas, no llegas a engatusarte. Pero si no sabes de qué va, nunca lo has visto en una relación con una chica pues es cuando caes (Grupo de discusión con chicas)".

En esta respuesta podemos observar cómo se utilizan algunas emisiones como "chulitos, interesantes, que te fijas en ellos" que suscitan en el receptor cierta curiosidad, que llaman la atención sobre lo que se está hablando. De hecho, se incluyen términos relacionados con el interés y con fijarse en alguien. La emisora deja manifiestamente claro en su comunicación verbal que los chicos que responden a ese perfil, son atractivos: "es cuando caes". La entonación del discurso en su relato y en sus gestos al realizar estas emisiones, constataba que ese acto comunicativo estaba impregnado de lenguaje del deseo hacia ese perfil de chico.

Sin embargo, a menudo cuando los actos comunicativos se utilizan para describir personas que tratan bien y no poseen una actitud o comportamiento violento o dominante no se utiliza el lenguaje del deseo, y de esta forma se les quita todo tipo de atractivo. En este ejemplo, un grupo de amigas hablan sobre un chico al que consideran buena persona pero no utilizan términos que transmitan deseo en su forma de expresarse:

"Sí. Bueno, majo, majo físicamente no lo es mucho, pero psíquicamente sí, sí es muy majo. Bueno y han acabado juntos!" (Relato comunicativo a chica).

En otras ocasiones se observa claramente la dualidad de estos dos tipos de lenguaje, del lenguaje del deseo y de la ética, donde se reproduce la doble moral tradicional. Por un lado se dota de atractivo y excitación relaciones donde se mantiene algún tipo de contacto afectivo-sexual de forma esporádica, en cambio, para iniciar una relación de pareja se asocia a la estabilidad y la 
seguridad utilizando un lenguaje de la ética que no resulta atractivo. Esta articulación implica fomentar unos actos comunicativos donde se vincula exclusivamente excitación y atractivo a un tipo de relación, mientras que el otro tipo queda exento de estos actos. De esta manera se va reproduciendo unas formas de hablar y expresarse en las y los jóvenes y adolescentes a través de las que se quita atractivo y excitación el inicio de una relación estable.

"R: Pues que sea un rollo de una noche que sea guapo y que esté bueno y ya está. Y que no sea... que no se drogue ni nada. $Y$ un novio que sea buena persona. $Y$ luego pues claro, si puede ser guapo pues mejor, si puede estar bueno pues mejor, pero que sea buena persona y ya está" (Relato comunicativo a chica).

La utilización del lenguaje de la ética también puede contribuir a perpetuar la no atracción hacia un modelo de atractivo igualitario. Esto ocurre cuando, por ejemplo, para hablar de personas que tratan mal, se utiliza el lenguaje de la ética diciendo que es una persona que no conviene. No se elimina el lenguaje del deseo donde se transmite que esa persona es excitante y deseable. Este lenguaje de la ética de persona 'no conveniente', persona 'no buena', tiene unos efectos en chicos y chicas: el aumento del deseo y atracción hacia los modelos de atractivo dominantes $\mathrm{y} / \mathrm{o}$ violentos.

“¿Pero por otro lado, cuando estaban juntos ella quería estar con él aunque tuviera estas presiones?

A: Creo que sí

B: Sí, porque nosotras le decíamos y por qué no lo dejas y ella quería seguir con él. ¿Ella que os explicaba de él cuando decidía seguir? ¿Os explicaba las presiones que tenía? ¿Qué os decía de por qué no lo dejaba?

B: Porque lo quería y porque ella quería estar con él.

A: Le daba igual. Pero es como los chicos de los que hablábamos antes, quiere tener uno y tener varios. Ese como era así de bueno, de 'tontín' siempre lo iba a tener ahí y tener a los demás, hasta que se dio cuenta de que no era tan tonto como ella pensaba. Luego se quedó un poco parada, pero no lo dejó" (Grupo de discusión con chicas).

\subsection{Actos comunicativos de poder}

La investigación ha detectado que las y los jóvenes utilizan actos comunicativos de poder que les conducen a mantener relaciones sin libertad y basadas en la dominación. Estos actos se van articulando de diferente forma: a) a través de las interacciones de poder que se mantienen en el grupo de iguales, b) del estatus social que los propios chicos y chicas otorgan a determinadas personas $\mathrm{y}, \mathrm{c}$ ) de la presión social existente para mantener determinadas relaciones o escoger personas concretas. 
a) Interacciones de poder

Aquellas personas que no poseen un estatus de prestigio dentro del grupo a menudo son excluidas en diferentes espacios del centro educativo. Esta exclusión se concreta en las interacciones entre iguales donde se van definiendo actos comunicativos de poder basados en pretensiones de poder cuyos efectos directos son la degradación de la imagen de las personas que los reciben.

“¿Le llamaban gorda? ¿Algo más fuerte le decían?

Sí, que no tenía formas, que era amorfa, que no... nadie (hacía nada)... ¿Ella cómo reaccionaba?

Bueno, ella pasaba" (Grupo de discusión con chicos).

Las interacciones en el grupo de iguales también tienen una gran influencia en la definición de los modelos de atractivo y las relaciones afectivo-sexuales. De hecho estas interacciones marcan de forma clara qué tipo de chicos y chicas son los que tienen un mayor éxito y, en consecuencia, un mejor estatus en el grupo. En determinados casos, los chicos utilizan actos comunicativos de poder que degradan la imagen de la mujer y la equiparan a un objeto sexual. Estos comentarios no desprestigian al chico en su entorno, todo lo contrario, ellos son conscientes de que les otorga mejor fama y por eso presumen de ello.

"Y aún así ¿tú crees que sigue gustando tanto a las chicas?

Pues no sé, él siempre habla de chicas de fuera que no conocemos nosotras.

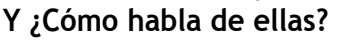

Te da muchos detalles de lo que hacen, que no es necesario. Así como presumiendo de lo que ha hecho en tema sexual.

¿Pero habla bien de las chicas o habla mal?

Habla más como si fueran un objeto, como esta me ha hecho esto y yo le he hecho esto" (Relato comunicativo a chica).

Estos comentarios que se dan entre los chicos, potencian su atractivo y se trata de chicos que ejercen el dominio sobre otras personas y que muestran públicamente esta actitud. De esta forma se va perpetuando un deseo que se va forjando a través de unos actos comunicativos de poder. Un ejemplo se observa en algunas relaciones donde las chicas se acostumbran a que los chicos las controlen y no les dejen salir con sus amigas, sin que ello implique una pérdida de interés por parte de las chicas.

"Pero luego empezó a ser muy posesivo, la llamaba a todas horas para saber dónde estaba, no la dejaba salir de casa, solo podía salir con él. La ropa que se ponía él le decía que no" (Grupo de discusión con chicas). 


\section{b) Estatus social}

Los y las adolescentes otorgan cierto estatus a determinados chicos y chicas. Se trata de aquellos y aquellas que son más populares y con los que la mayoría de la gente quiere mantener relaciones afectivo-sexuales. Este estatus se va articulando a través de actos comunicativos que los y las jóvenes definen en el grupo de iguales a partir de un lenguaje del deseo que vincula dominación y desprecio con atracción. De esta forma se generan situaciones fundamentadas en pretensiones de poder en las que los chicos y chicas basan su elección en la escala social que ocupa la persona que escogen.

Los chicos y las chicas se dejan guiar por lo que dice la gente.

"Pues a ver, este tío ha salido con muchísima gente, a ver, vamos a probar, pues a ver si es verdad y si ves que es mejor quedas con él. Pero si dicen este tío es un 'pringao' y no ha estado nunca con nadie, pues ya pasas directamente porque... no haber estado con nadie puede parecer malo para alguna gente" (Grupo de discusión con chicos).

Existen determinados contextos donde las y los adolescentes establecen numerosas interacciones en torno a sus relaciones afectivo-sexuales. Cuando los actos comunicativos de poder predominan en estos contextos, como pueden ser las discotecas o las redes virtuales, los chicos y chicas van articulando relaciones basadas en el engaño de forma que no existe ni libertad ni consenso. En esas situaciones se propician interacciones de poder, que basadas en el engaño y en la falta de sinceridad, dotan de mayor atractivo al chico en cuestión.

“¿Tú crees que cuando los tíos quieren enrollarse con una tía, les mienten o son sinceros?

Para una noche seguro que les mienten.

¿Qué tipo de mentiras crees que pueden decir?

Pues la... la edad, seguro.

¿En qué, se ponen más o menos?

Sí, hombre, bien más.

¿Porque así ganan valor?

Claro. Yo qué sé. A lo mejor hay un tío de diecisiete y se coge a una de diecinueve y le dice "tengo veintidós'” (Relato comunicativo a chica).

Por otro lado, los actos comunicativos que se definen en los entornos virtuales también conducen a situaciones parecidas. Los actos comunicativos que se desarrollan en redes sociales como Facebook, Fotolog y Messenger se basan en muchos casos en pretensiones de poder que consiguen efectos concretos en las relaciones de los y las adolescentes. Por ejemplo en algunas ocasiones en estos espacios se aprovecha para vender una imagen no real de uno o una misma 
que persigue contribuir a mejorar el propio estatus social. La siguiente cita muestra cómo algunos chicos utilizan estas herramientas para dar una imagen de sí mismos que no es real.

"B: Pues más prepotente, porque como no saben cómo es en la vida real, se crece, hace como su fuera un 'superhombre'. Se crea una personalidad que no es la suya y se cree superior a todos los demás. Porque dice que es el mejor en los deportes, en las notas, en todo" (Grupo de discusión a chicos).

\section{c) Presión social}

En determinados casos en los actos comunicativos se pueden dar elementos que contribuyan a desencadenar presión social en la toma de determinadas decisiones, o reproducir la exclusión de aquellas personas que no tienen un estatus social elevado dentro del grupo de iguales. En el primer caso las consecuencias pueden llevar a empezar relaciones basadas en la libertad o carentes de ella. En el segundo la presión social puede generar una cadena de menosprecio hacia las personas con un menor estatus. Con relación a este último punto, en alguna de las situaciones explicadas por los y las adolescentes se aprecia claramente cómo, por miedo a ser rechazado o rechazada por los compañeros y compañeras, no se evitan comentarios despectivos que humillan a otras personas.

“(...) A veces sí, pero, se dice: ay pues, para que no me separen, claro pues no me marginen, para que no piensen mal de mí y sigo el rollo como mis amigas" (Relato comunicativo a chica).

En otras situaciones esta presión, y como se transmite a través de los comentarios del grupo de iguales, tiene un impacto muy directo en la vida de los chicos y chicas. En este sentido a menudo ellos y ellas sienten que deben empezar una relación aunque no lo deseen. Existe de alguna forma una coacción aunque se articule de forma no explícita ya que viene definida por determinados procesos de socialización de las chicas y los chicos.

"Hombre, supongo que un poco sí, ¿no?, porque claro, ya las otras empiezan a hablar, que si ha estado con este o con aquel, y a lo mejor pues esa chica se siente un poco frustrada, porque claro, ella no tiene experiencia en eso ni nada" (Relato comunicativo a chica).

De manera que de forma estructural esta presión se va consolidando en la vida afectiva y sexual de los chicos y chicas. Lo podemos observar en la siguiente cita donde una chica decide iniciar una relación con un chico sin que fuera su deseo explícito. El hecho de que sus compañeras tuvieran pareja o una relación esa noche desencadenó esta situación.

"Si en un concierto de aquí, cerca, una chica no quería salir con el chico porque le gustaba 
otro, pero al ver que todas las chicas tenían un novio o algo, un chico vino la cogió y le dijo de hacer una vuelta y tal, y la chica acabó con él, pero no quería, la verdad" (Grupo de discusión con chicos).

\subsection{Actos comunicativos dialógicos y solidaridad}

Finalmente cabe señalar, en contraposición a los anteriores componentes presentados hasta ahora, que existen actos comunicativos que tienen una repercusión positiva en la superación de las situaciones de abuso o violencia de género. La investigación ha permitido identificar que existen actos comunicativos dialógicos e interacciones forjadas en la solidaridad y el consenso que se concretan en el grupo de iguales, concretamente en los grupos de chicas, que están ayudando a que algunas de ellas dejen relaciones marcadas por el maltrato.

"Le hicimos ver de alguna manera que no podía seguir así, que ya tenía que estar harta de maltratos tanto psicológicos como físicos, que se merecía vivir con ella, que ya no era por él, si no por ella misma y bueno, quizás le comimos mucho la cabeza pero fue la única manera de que realmente se diera cuenta" (Relato comunicativo a chica).

Estos actos comunicativos promueven unas relaciones afectivo-sexuales más satisfactorias ya que favorecen que los chicos y chicas puedan escoger con mayor libertad que tipo de relación quieren iniciar y con quién. Potenciar estos actos en los centros escolares puede consolidar la construcción de relaciones igualitarias. Es importante que se definan a través de un lenguaje del deseo que relacione atractivo y deseo con igualdad y solidaridad. La investigación ha identificado que los comentarios de los amigos y las amigas sobre estos temas está siendo una de las fórmulas para favorecer este tipo de relaciones.

\section{CONCLUSIONES}

Casos como los de Phoebe en Estados Unidos y en otros países ponen de manifiesto la necesidad de estudios científicos que profundicen sobre las causas de la violencia de género entre adolescentes. La concepción de los actos comunicativos aporta elementos para ello. Por ejemplo: las interacciones en el grupo de iguales jugaron un papel importante en las situaciones de acoso que vivió Phoebe, al igual que el estatus social que determinadas personas de su entorno poseían y el lenguaje que utilizaban para desprestigiarla e humillarla así como los mensajes que se elaboraron en la red Facebook.

Los resultados presentados han permitido analizar los actos comunicativos que utilizan las y los adolescentes y jóvenes cuando hacen referencia a sus relaciones afectivo-sexuales. A través de este análisis ha sido posible identificar aquellos actos comunicativos que promueven relaciones 
basadas en la dominación y la no libertad y aquellos que favorecen relaciones libres e igualitarias. En este sentido se ha constatado, sobre todo, que el tipo de lenguaje que utilizan los y las adolescentes en sus interacciones cotidianas en los centros educativos es un elemento que permite conocer con mayor detalle cómo se configuran estas relaciones. Por otro lado, también la estructura social, como hemos podido observar, se convierte en un componente esencial ya que tiene una incidencia directa en la configuración de los propios actos.

Algunas experiencias educativas y sociales que están trabajando para la superación de la discriminación de género o para la prevención de la violencia machista se han hecho eco de estos análisis y los están utilizando con éxito en su práctica o lucha cotidiana (Oliver, Soler \& Flecha, 2009; Valls et al. 2008). En este sentido, seguir con esta transferencia entre el conocimiento científico y la sociedad civil está promoviendo el avance hacia relaciones cada vez más forjadas en actos comunicativos dialógicos. De esta manera es posible dar pasos para prevenir y erradicar casos como los de Phoebe en todo el mundo.

\section{REFERENCIAS BIBLIOGRÁFICAS}

Alandete, D. (2010). En manos de las chicas malas. El País.

Austin, J. (1975). How to do things with words. Cambridge: Harvard University Press.

Boeringer, S. (1999). Associations of rape-supportive attitudes with fraternal and athletic participation. Violence Against Women, 5(1), 81-90.

Connell, R. (2005). Masculinities. Cambridge: Polity Press.

CREA (Centre of Research in Theories and Practices that Overcome Inequalities). (2006-2008). Actos comunicativos y superación de las desigualdades sociales en las relaciones de género. Plan Nacional I+D+I, Universidad de Barcelona.

CREA. (2006-2011). INCLUD-ED. Strategies for inclusion and social cohesion from education in Europe. Comunidad Europea Commission, Universidad de Barcelona.

CREA. (2008). Literature on effective educational practices in Europe. Gender. INCLUD-ED. strategies for inclusion and social cohesion from education in Europe, Universidad de Barcelona.

Duque, E. (2006). Aprendiendo para el amor o para la violencia. Las relaciones en las discotecas. Barcelona: Hipatia.

Flecha, R. \& Gómez, J. (2004). Participatory paradigms: Researching ‘with' rather than 'on'. En B. Crossan, J. Gallacher \& M. Osborne (Eds.), Researching widening access: Issues and approaches in an international context (pp. 129-140). London: Routledge.

Flecha, R. (2008). Aprendizaje dialógico: Creación de sentido. Congreso de Comunidades de Aprendizaje. Gobierno Vasco, Departamento de Educación, Bilbao, España. 
Giordano, P., Longmore, M. \& Manning, W. (2006). Gender and the meanings of adolescent romantic relationships: Focus on boys. Americal Sociological Review, 71(2), 260-287.

Gómez, J. (2004). El amor en la sociedad del riesgo. Barcelona: Hipatia.

Habermas, J. (1984). The theory of communicative action. V1. Reason and the rationalization of society. Boston: Beacon Press.

Hage, S. (2006). Profiles of women survivors: The development of agency in abusive relationships. Journal of Counseling \& Development, 84, 83-94.

Instituto de la Mujer. (2006). III Macroencuesta. Violencia contra las mujeres [en línea]. Disponible en: http://www.inmujer.migualdad.es/mujer/mujeres/estud_inves/violencia\%20 final.pdf

Instituto de la Mujer. (2010). Datos violencia de género. Año 2009. Madrid: Instituto de la Mujer.

Klein, J. (2006). An invisible problem - everyday violence against girls in schools. Theoretical Criminology, 10(2), 147-177.

MacGeorge, E., Graves, A., Bo Feng, G. \& Burleson, B. (2004). The myth of gender cultures: Similarities outweigh differences in men's and women's provision of and responses to supportive communication. Sex Roles, 40(3-4), 143-175.

Oliver, E., Soler, M. \& Flecha, R. (2009). Opening schools to all (women): Efforts to overcome gender violence in Spain. British Journal of Sociology of Education, 30(2), 207-218.

Puigvert, L. (2008). Coeducación en siglo XXI: Lenguaje de los valores y lenguaje del deseo. V Jornadas Provinciales de Coeducación: Interculturalidad desde la perspectiva de género, Granada, España.

Searle, J. (1999). Mind, language and society. New York: Basic Books.

Soler, M. \& Ramis, M. (2008). Freedom in gender relations. An analysis of communicative acts among adolescents. First ISA Forum. International Sociological Association. Barcelona, España.

Stobbe, L. (2005). Doing machismo: Legitimating speech acts as a selection discourse. Gender work and Organization, 12(2), 105-123.

Valls, R., Puigvert, L. \& Duque, E. (2008). Gender violence among teenagers: Socialization and prevention. Violence Against Women, 14(7), 759-785.

\section{NOTAS}

1 Los proyectos I+D+I son aquellos proyectos competitivos con mayor prestigio científico en España que son financiados por el Ministerio de Ciencia e Innovación. 